\title{
ARTICLE
}

\section{Mitigation of radiation streaming inside the ITER ports}

\author{
Arkady Serikov ${ }^{a^{*}}$, Ulrich Fischer ${ }^{\mathrm{a}}$, Mark Henderson ${ }^{\mathrm{b}}$, Dieter Leichtle ${ }^{\mathrm{a}}$, Charles Spencer Pitcher ${ }^{\mathrm{b}}$, Peter Spaeh ${ }^{\mathrm{a}}$, Dirk \\ Strauss $^{\mathrm{a}}$, Alejandro Suarez ${ }^{\mathrm{b}}$ and Bastian Weinhorst ${ }^{\mathrm{a}}$ \\ ${ }^{a}$ Karlsruhe Institute of Technology (KIT), Institute for Neutron Physics and Reactor Technology, Hermann-von-Helmholtz-Platz 1 , \\ 76344 Eggenstein-Leopoldshafen, Germany; ${ }^{b}$ ITER Organization, Route de Vinon sur Verdon, 13115, St. Paul lez Durance, \\ France
}

This paper concerns the ITER project demands to provide the machine reliability and the human safety along and after the ITER operation for making the personnel access inside the ITER bioshield possible. New results are presented for radiation streaming inside the gaps of Diagnostic Equatorial Port Plug (EPP) of ITER with estimation of cross-leakage from over ITER components and consequence of such leakage to upper port with embedded Electron Cyclotron Heating Upper Launcher (ECHUL). It was shown that neutron and photon streaming dominates the radiation environment in the interspace areas at the rear-sides of the ports where personnel access is planning. Several methods to mitigate that adverse radiation streaming are elaborated, with a well considered parametric analysis to reduce gap streaming in EPP by means of different labyrinth configurations, and rails inside the EPP gaps. State-of-the-art codes were applied in the analysis: radiation transport with MCNP5, activation inventory calculations with FISPACT-2007, and interface code R2Smesh to couple transport with activation by using common mesh-tally to produce shut-down dose rate maps with high resolution and fidelity. The mitigation of neutron streaming inside the ports is achieved by understanding of the neutron/photon pathways in radiation transport phenomenon, revealing the dominant radioisotopes, and foundation a strategy for radiation shielding optimization for the ITER ports.

Keywords: ITER; fusion neutronics; diagnostics; equatorial port; upper port ECH launcher; shut-down dose rate; MCNP; R2Smesh

\section{Introduction}

A phenomenon of forward biased anisotropy of neutron scattering is an intrinsic feature of the angular distribution of D-T $14 \mathrm{MeV}$ source neutrons impinging on the ITER First Wall (FW). An example of forward biased angular distribution of neutrons incident on the FW of the ITER blanket module 13 is presented in [1]. The D-T pointwise neutron source was defined in the plasma shown in Figure 1 as a matrix of neutron born probabilities corresponded to $500 \mathrm{MW}$ of fusion power [2]. The module 13 is located above the Equatorial Port Plug (EPP) depicted in vertical cut of the 3D neutronics model in Figure 1, where the highest value of neutron wall load is expected for the whole poloidal distribution on the ITER blanket FW [2]. These prerequisites constitute conditions for increased neutron streaming through any of FW openings of the Diagnostic Equatorial Port Plug (EPP), and currently computational neutronics support is providing for its engineering design development to arrange optimal shielding and mitigate harmful streaming effects. In case of straight gaps with direct line-of-sight to the plasma, this effect

*Corresponding author. Email: arkady.serikov@kit.edu causes high activation of the materials surrounded the EPP gaps shown in Figure 2 and results in excessively high Shut-Down Dose Rate (SDDR) inside the interspace of Port Extension (PE). According to the ITER nuclear safety regulations for areas with personal access, the target value of SDDR is $100 \mu \mathrm{Sv} / \mathrm{h}$ at $10^{6} \mathrm{~s}$ after shut-down [2]. Neutron streaming results in activation of the steel materials of the EPP closure plate and the port flange, shown in Figure 2. Activation analysis [3] revealed the following dominant radioisotopes: ${ }^{60} \mathrm{Co},{ }^{182} \mathrm{Ta},{ }^{58} \mathrm{Co},{ }^{54} \mathrm{Mn}$, and ${ }^{59} \mathrm{Fe}$.

The diagnostic equipment is supposed to be packed inside the three vertical diagnostic shield module (DSM) drawers are separated in the toroidal direction by narrow inter-drawer gaps $(\sim 5 \mathrm{~mm})$. The DSM drawers are shown in Figure 3. Such separation provides an assembly tolerance and cuts eddy currents induced during plasma disruptions.

To minimize the EPP material activation and subsequent SDDR in the PE-interspace, the streaming pathways were examined carefully in a dedicated parametric shielding and activation analyses [3] using the Monte Carlo approach with specially adapted local EPP models. As an outcome of the parametric analyses, 
a double-labyrinth EPP design has been recommended for integration into the global neutronics model of ITER, and verification [4] has shown validity of the local model approach for quick SDDR assessments. In this paper, corrections of SDDR have been introduced in relation with leakages from gaps and voids inside other ITER components.



Figure 1. Vertical cut of the ITER 3D MCNP neutronics model (B-lite) with ECHUL. The five interspace control volumes F1 to F5 are used for tallying the neutron flux.

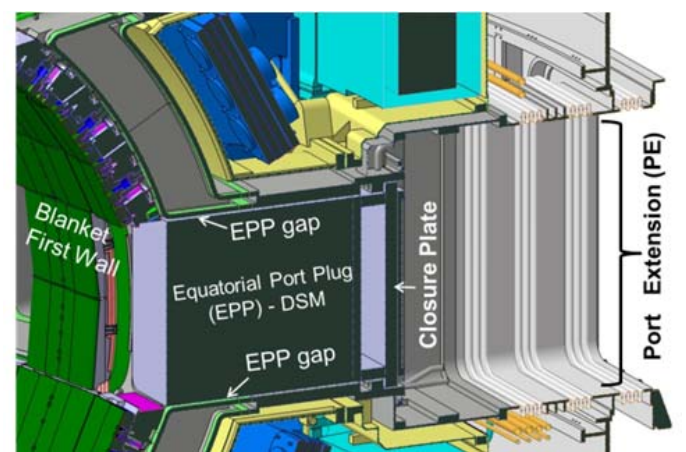

Figure 2. Vertical cut through the Computer Aided Design (CAD) model of the Equatorial Port.

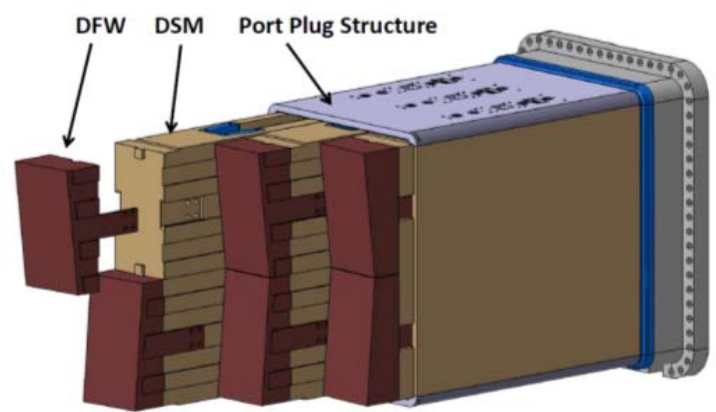

Figure 3. CAD view of Equatorial Port Plug (EPP) showing the assembly of Diagnostics FW to the DSM, then insertion the DSM drawers into the port plug structure.

Another object for analysis is the Electron Cyclotron Heating Upper Launcher (ECHUL) to be installed inside the ITER upper port, see Figure 1. Upper port is inclined above the plasma core, and hence the neutron load on FW of ECHUL is less intense. The main issue concerning ECHUL is to hinder radiation penetration from the Blanket Shield Module (BSM), because BSM is substantially voided due to the need to open the FW for injection of the mm-waves entering to the plasma from the upper port launcher [5]. The long-term radiation shielding support for the ECHUL development confirmed its capability to withstand neutron damage and nuclear heating for the mm-wave focusing and steering mirrors located close to the plasma opening [5].

\section{Methodological approach}

The needs of the neutronics based engineering design development of EPP have requested to conduct SDDR estimations to check with SDDR target of $100 \mu \mathrm{Sv} / \mathrm{h}$ at $10^{6} \mathrm{~s}$ after shut-down for personal access. These needs were satisfied by using the Monte Carlo code MCNP5 [6] and reference 3D ITER model B-lite shown in Figure 1 with the FENDL-2.1 neutron cross-section library. Activation calculations have been performed with the FISPACT-2007 [7] inventory code with corresponding EAF-2007 activation data file. In order to obtain the SDDR results, we have coupled transport and activation calculations by means of the R2Smesh interface system [8] through the MCNP-FISPACT common use of a mesh superimposed over the MCNP geometry.

Logically, methodological approach includes three major stages: on the $1^{\text {st }}$ stage, exhaustive neutronics parametric (geometry and materials) analyses were performed in local MCNP models as it was elaborated in [3]. The $2^{\text {nd }}$ stage was devoted to verification and correction of the SDDR results by integration of them into the global ITER model [4]. On the $3^{\text {rd }}$ stage, the mutual influence of ITER components was investigated in sense of radiation cross-leakage from one component to another, and in this paper the cross-leakages from several gaps in three ITER ports, blanket and its manifold were estimated with an incremental approach.

\section{Results for diagnostics EPP}

As the result of neutronics parametric analyses of the EPP gap labyrinth configuration by scanning its length and shift [3, 4], an optimized double labyrinth variant shown in Figure 4 has been chosen for mitigation the streaming inside the EPP gaps. Then, this EPP double labyrinth configuration has been embedded into the global MCNP model B-lite, with the resulting map of SDDR displayed in Figure 5 with SDDR of $58 \mu \mathrm{Sv} / \mathrm{h}$ averaged over the control volume F1 of EPP. The port plug is composed with $80 \%$ of steel and $20 \%$ of water and does not include any diagnostic penetrations, which contribution to whole SDDR is assumed at the first approximation to be the half of $100 \mu \mathrm{Sv} / \mathrm{h}$ access limit. That means target value of SDDR inside the port interspace with homogeneous plug is $50 \mu \mathrm{Sv} / \mathrm{h}$. 


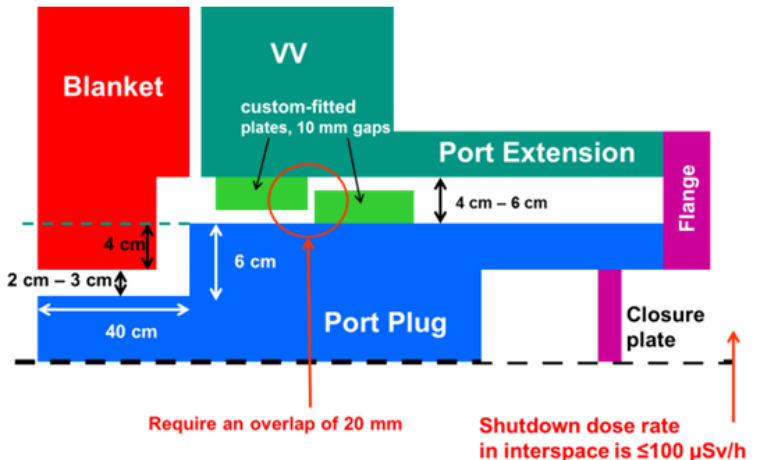

Figure 4. Schematic view of the optimized double labyrinth EPP gap configuration (vertical cut).

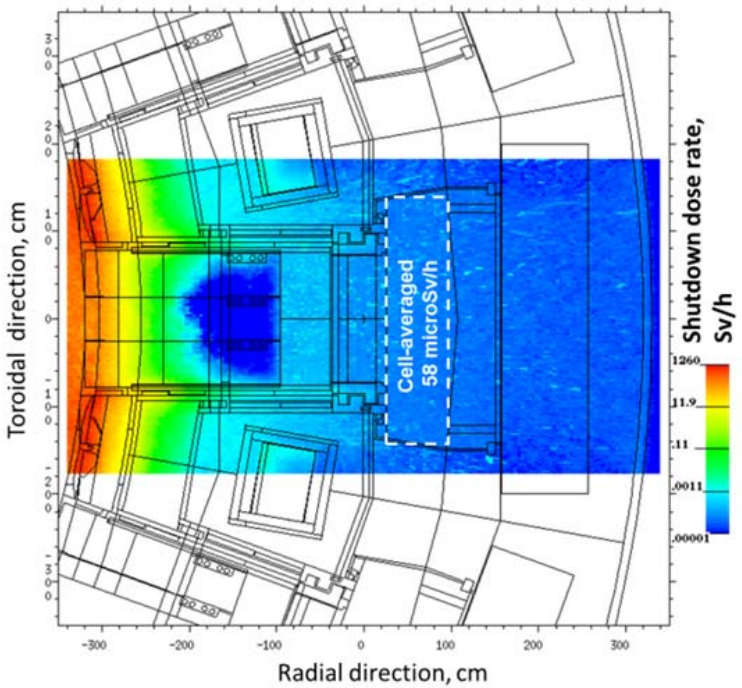

Figure 5. Map of shut-down dose rate (SDDR) in the vicinity of the Equatorial Port Plug -EPP (with closed upper and lower ports) B-lite MCNP model horizontal cut.

The map in Figure 5 illustrates radiation streaming along the 4-cm all-round EPP gaps, but in the middle of DSM EPP the SDDR is very low because this EPP model does not include the 0.5 -cm inter-drawer gaps. As it was indicated in early estimates [3, 4], straight gap shown in Figure 6A allowing direct line-of-sight to the plasma should be excluded because of extremely strong streaming, as it is illustrated in Figure 7. To mitigate streaming inside the inter-drawer gaps, the horizontal bars of the rails are arranged inside the DSM inter-drawer 0.5-cm gaps, with a configuration shown in Figure 6B and flux attenuation inside the gaps with rails - in Figure 7.

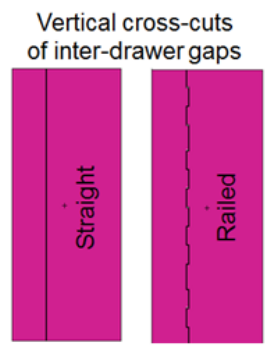

Figure 6A. EPP inter- drawer gap shapes.

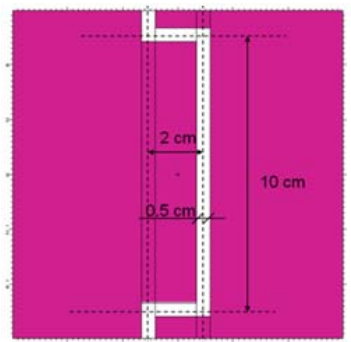

Figure 6B. Railed gap configuration.

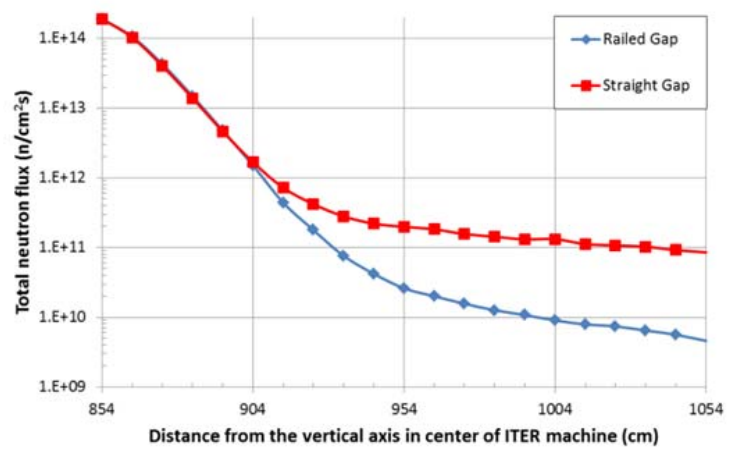

Figure 7. Comparison of total neutron flux attenuation inside the straight and rail-shaped inter-drawer gaps of DSM EPP.

Comparison of total neutron flux curves plotted in Figure 7 for the railed and straight gaps allows to conclude that the rail-shaped inter-drawer gaps provide the additional attenuation of the total neutron flux at the back of the drawer of the order of 10 . The reason for such stronger mitigation of the streaming is related to the splitting of the solid angle by which the detector inside the EPP F1-interspace is covered in the poloidal direction of the plasma source. In case of gaps with rails, neutrons encounter more nuclear interactions with the steel material of the rails. Rails inside the DSM gaps are effective for DSM thicker than $50 \mathrm{~cm}$, in case of moderated and low energy neutrons. Streaming through the inter-drawer gaps in the middle of DSM is suppressed by rails. That is quite noticeable in Figure 8 in comparison with much stronger streaming through the straight 4-cm EPP gaps on the lateral sides of EPP DSM.

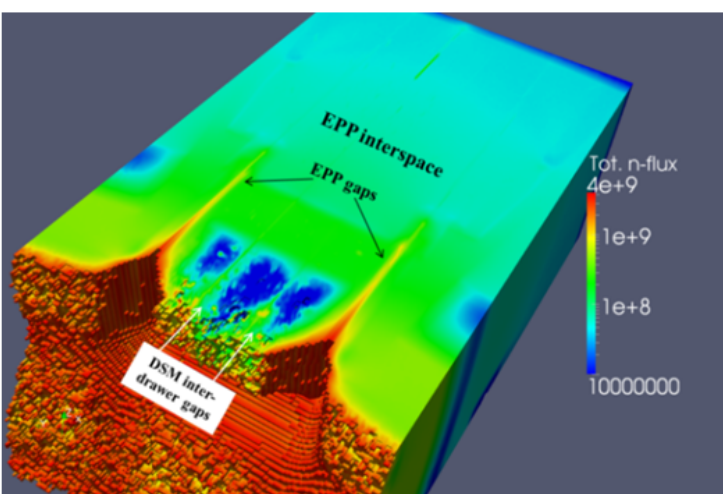

Figure 8. Map of total neutron flux $\left(\mathrm{n} / \mathrm{cm}^{2} / \mathrm{s}\right)$ for B-lite model with railed inter-drawer gaps inside DSM.

The work strategy persuaded the ultimate aim to mitigate gap streaming in ITER ports. The realization was fulfilled by quantification the streaming effects inside the EPP and leakages from other ITER components to the SDDR inside the EPP control volume F1 pinpointed in Figure 1. This strategy follows to an incremental approach of subsequent opening the gaps inside the EPP and other gaps in ITER parts from which neutron and gamma radiation causes increase of SDDR inside the F1 interspace, as illustrated in Figure 9. 


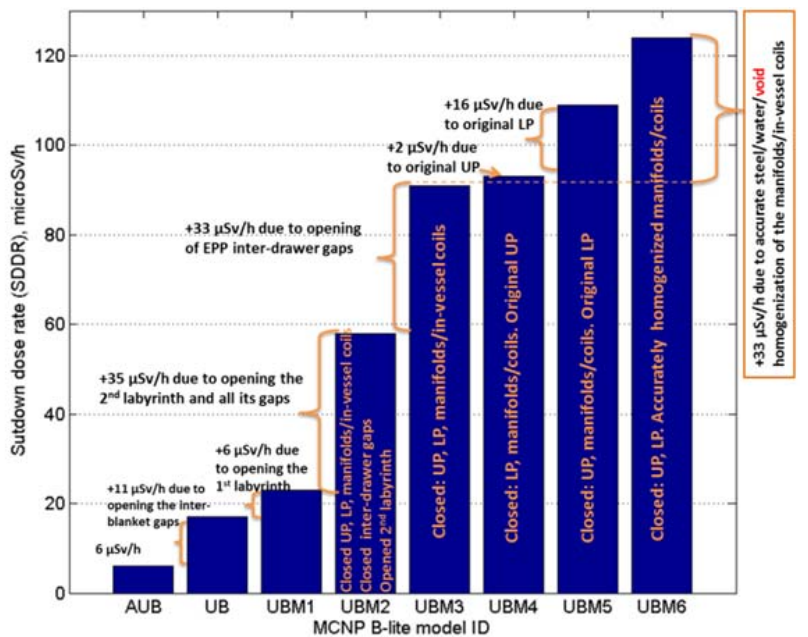

Figure 9. Incremental approach for the SDDR estimation inside the F1 interspace of EPP due to subsequent gaps opening in the MCNP B-lite model.

The increments of SDDR in histogram of Figure 9 were calculated in separate MCNP models with IDs ranging from AUB to UBM6. It is started from the most shielded B-lite model's ID called AUB (Absolute Ultimate Baseline) in which every gap inside the outboard blanket and three ports are closed with a shielding material composed of $80 \%$ of steel and $20 \%$ of water. The first dose increment of $11 \mu \mathrm{Sv} / \mathrm{h}$ is caused by opening the gaps between the blanket modules in the UB model. Additional $6 \mu \mathrm{Sv} / \mathrm{h}$ has contributed to the F1 control volume due to opening the $1^{\text {st }}$ labyrinth of the EPP double-labyrinth in the Ultimate Baseline Modification 1 (UBM1) model. Next increment is related to opening of the $2^{\text {nd }}$ labyrinth, depositing $35 \mu \mathrm{Sv} / \mathrm{h}$ to $\mathrm{F} 1$ of the model UBM2. Then, railed inter-drawer gaps in model UBM3 result in increase in the SDDR inside $\mathrm{F} 1$ on $33 \mu \mathrm{Sv} / \mathrm{h}$. It is remarkable that this $33 \mu \mathrm{Sv} / \mathrm{h}$ increment in UBM3 model is equivalent to the dose deposition in model UBM6 caused by the accurate modelling of the pockets of blanket manifolds and in-vessel coils with homogeneous steel/water/void mixtures calculated based on 3D CAD files, in which void fraction varied reaching up to 70 vol.\%. On contrary, in all the previous models in a range of AUB UBM5 these pockets were closed by $80 \%-20 \%$ steel-water shielding. Neutronics modelling assumptions applied for the pockets of blanket manifolds and in-vessel coils were shown in [9] to significantly affect fluxes and doses in both upper and equatorial port interspaces, as follows from neutronic analyses of the Electron Cyclotron Heating Upper Launcher (ECHUL) integrated into different B-lite variants [9].

Excessive neutron leakage and elevated radiation levels in one interspace volume will influence the level at neighbouring locations, in particularly due to the streaming in the original Upper Port (UP) in UBM4 and original Lower Port (LP) in UBM5, there are increases of SDDR pointed in Figure 9.

The SDDR results have been provided at $1 \mathrm{e} 6 \mathrm{~s}$ after the ITER shutdown using the ICRP74 photon-to-dose conversion factors [10]. The SA2 neutron irradiation scenario [11] has been applied for the FISPACT part of the R2Smesh interface; it assumes $0.3 \mathrm{MWa} / \mathrm{m}^{2}$ of neutron fluence on the FW of all in-vessel components, in accordance with [2]. The SDDR results are based on the generally reliable statistical quality of the MCNP5 results with less than $10 \%$ of relative statistical errors.

\section{Conclusion}

The problem of excessive neutron streaming through the Equatorial Port Plug gaps has been alleviated by means of arranging the suitable labyrinths devised as the results of the parametric neutronics analysis. Direct line-of-sight streaming from the plasma should be avoided. An incremental approach has been applied quantifying the gap and void effects to the shut-down dose rate inside EPP interspace. It allows measuring the leakages caused by cutouts in blanket meant for blanket manifolds and in-vessel coils which affect both upper and equatorial port interspaces. As a major result of the analyses performed, an optimized double labyrinth around EPP was recommended for implementation.

\section{Acknowledgements}

This work has been funded mainly by the ITER Organization under the ITER service contracts Nr. IO/10/4300000298 and Nr. IO/12/4300000548 and partly by the European Joint Undertaking for ITER and the Development of Fusion Energy (Fusion for Energy) under contract No. F4E-2010-GRT-161 using the B-lite MCNP model which was developed as a collaborative effort between the FDS team of ASIPP China, University of Wisconsin-Madison, ENEA Frascati, CCFE UK, JAEA Naka, and the ITER Organization.

The views and opinions expressed herein do not necessarily reflect those of the ITER Organization.

\section{References}

[1] B. Smith, P. Wilson, M. Sawan and T. Bohm, Source profile analysis for the ITER first wall/ shield module 13, Fusion Science and Technology 56-1 (2009), pp. 57-62.

[2] H. Iida, V. Khripunov and Nuclear Analysis Group, Nuclear Analysis Report (NAR), ITER Naka \& Garching Joint Work Sites Internal Report G 73 DDD 2 W 0.2, ver. 2, (2004).

[3] A. Serikov, U. Fischer, D. Leichtle and C.S. Pitcher, Monte Carlo radiation shielding and activation analyses for the Diagnostic Equatorial Port Plug in ITER, Fusion Engineering and Design 87 (2012), pp. 690-694.

[4] A. Serikov, U. Fischer, D. Leichtle, C. S. Pitcher and A. Suarez, Verification of the local Monte Carlo modelling approach for fusion neutronics applications in ITER, Transactions of the American Nuclear Society 106 (2012), pp. 641-644. 
[5] A. Serikov, U. Fischer, D. Grosse, P. Spaeh and D. Strauss, Evolution of shielding computations for the ITER Upper ECH Launcher, Nuclear Technology 175 (1) (2011), pp. 238-250.

[6] X-5 Monte Carlo Team, MCNP-A General Monte Carlo N-Particle Transport Code, Version 5, Volume I, MCNP Overview and Theory, LA-UR-03-1987, Los Alamos National Laboratory Report, April 24, 2003.

[7] R. A. Forrest, FISPACT 2007 User Manual, UKAEA FUS 534 report, March 2007.

[8] M. Majerle, D. Leichtle, U. Fischer and A. Serikov, Verification and validation of the R2Smesh approach for the calculation of high resolution shutdown dose rate distributions, Fusion Engineering and Design 87 (2012), pp. 443-447.
[9] A. Serikov, U. Fischer, M. Henderson, D. Leichtle, C.S. Pitcher, P. Spaeh, D. Strauss, A. Suarez and B. Weinhorst, Neutronics for equatorial and upper ports in ITER, Proceedings of the 27th SOFT conference, Liege, Belgium, Sept. 24-28, 2012, Fusion Engineering and Design (2013), http://dx.doi.org/10.1016/j.fusengdes.2013.03.037.

[10] ICRP Publication 74, Conversion coefficients for use in radiological protection against external radiation, Ann. ICRP, vol. 26, no. 3/4, Elsevier, Amsterdam, 1996.

[11]M.J. Loughlin and N.P. Taylor, Recommended plasma scenarios for activation calculations, ITER Internal Report ITER_D_2V3V8G v 1.1 (2009). 